\title{
Insect-induced gene expression at the core of volatile terpene release in Medicago truncatula
}

\author{
Wayra G. Navia-Giné, ${ }^{1}$ S. Karen Gomez, ${ }^{1, \dagger}$ Joshua Yuan, ${ }^{2, \ddagger}$ Feng Chen ${ }^{2}$ and Kenneth L. Korth 1,* \\ 'Department of Plant Pathology; University of Arkansas; Fayetteville, AR USA; 2 Department of Plant Science; University of Tennessee; Knoxville, TN USA \\ †Present address: Boyce Thompson Institute; Ithaca, NY USA; łPresent address: Texas A\&M University; College Station, TX USA
}

Key words: legume, ocimene, terpene synthases, plant volatiles

The blends of induced volatiles released by higher plants in response to herbivory regularly contain terpenoids. The precursors of volatile terpenoids can be synthesized via two pathways, the mevalonate (MVA) and the methyl erythritol 4-phosphate (MEP) pathways localized in the cytosol and in plastids, respectively. Terpenes are important players in interactions between plants and herbivorous insects, by acting in both direct and indirect defenses. We recently characterized a gene encoding an $(E)$ - $\beta$-ocimene synthase (MtEBOS) in the legume Medicago truncatula Gaertn. Compared to undamaged plants, caterpillardamaged $M$. truncatula emitted $(E)$ - $\beta$-ocimene at an elevated level and this increase is associated with high levels of expression of MtEBOS mRNA. Exogenous treatment with jasmonic acid also increases transcript accumulation of $M t E B O S$. These results indicate that transcript accumulation is used as a tightly regulated mechanism to control $(E)$ - $\beta$-ocimene emission. The data, along with additional findings in other species, illustrate that like most plant families legumes regulate the final steps of volatile terpene biosynthesis at the level of transcript induction.

The barrel medic Medicago truncatula, like many other plant species, utilizes multiple mechanisms of defense to counteract pathogen and herbivore attack. Plant defense against herbivorous insects can be manifested by the release of volatile chemicals to attract natural enemies of the herbivores as indirect defense, or by production of compounds to harm the herbivore as a direct defense. ${ }^{1,2}$ The most common class of induced (formed de

\footnotetext{
${ }^{*}$ Correspondence to: Kenneth L. Korth; Department of Plant Pathology; 217 Plant Science Building; University of Arkansas; Fayetteville, AR 72701 USA; Tel.: 479.575.5191; Fax: 479.575.2771; Email: kkorth@uark.edu

Submitted: 05/07/09; Accepted: 05/07/09

Previously published online as a Plant Signaling \& Behavior E-publication: http://www.landesbioscience.com/journals/psb/article/8971

Addendum to: Navia-Giné WG, Yuan JS, Mauromoustakos A, Murphy JB, Chen F, Korth KL. Medicago truncatula (E)-B-ocimene synthase is induced by insect herbivory with corresponding increases in emission of volatile ocimene. Plant Physiol Biochem 2009; 47:416-25; PMID: 19249223; DOI: 10.1016/i.plaphy.2009.01.008.
}

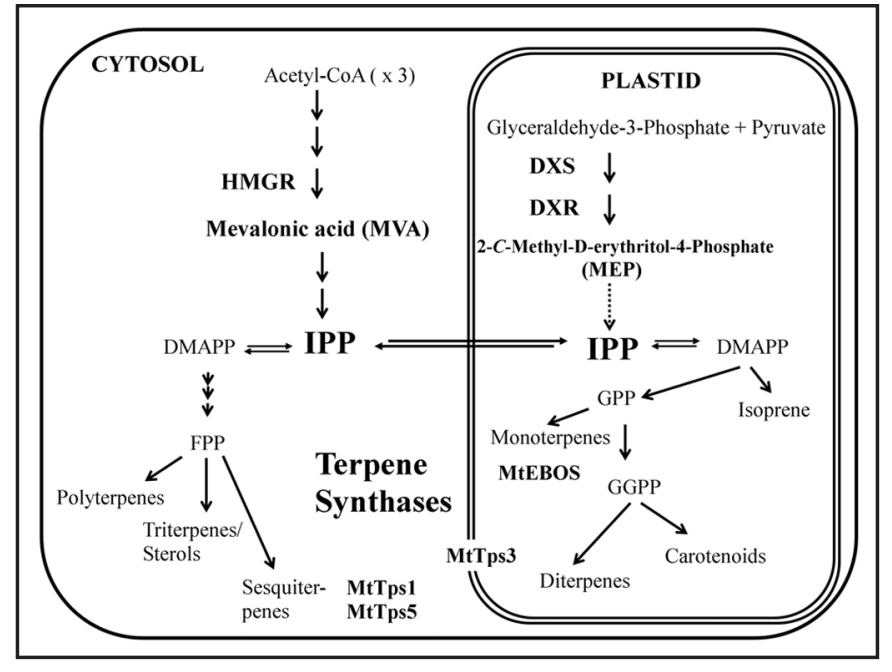

Figure 1. A model for biosynthesis of terpenes via the cytosolic mevalonate (MVA) pathway and the plastidic 2-C-methyl erythritol 4-phosphate (MEP) pathway, indicating that a high degree of pathway crosstalk can occur. Recent data indicate that the enzyme encoded by MtEBOS functions solely in the synthesis of monoterpenes, primarily $(E)$-?-ocimene. The indicated enzymes are: 3-hydroxy-3-methylglutaryl CoA reductase (HMGR), 1-deoxy-D-xylulose-5-phosphate synthase (DXS), 1-deoxy-D-xylulose-5phosphate reductoisomerase (DXR) and individual terpene synthases (TPS). The indicated precursors are: isopentenyl diphosphate (IPP), dimethyl allyl diphosphate (DMAPP), farnesyl pyrophosphate (FPP), geranyl diphosphate (GPP) and geranylgeranyl diphosphate (GGPP). The dashed arrow represents more than one step (figure adapted from Bede et al. 2006).

novo) plant volatiles are the terpenes, which play an important role during indirect defenses. ${ }^{3,4}$ Other classes of compounds, such as short-chain alcohols and aldehydes, are also commonly released from injured plants. Volatile blends can be specific to the insect species ${ }^{5}$ or to the insect developmental stage ${ }^{6}$ causing the damage, and the quality and quantity of volatile blends from a given plant species is largely determined by degree of damage and insect-feeding behavior. ${ }^{7}$ Regulation of production of the incredibly diverse range of terpenes is controlled at several levels, as compounds are produced from the five-carbon precursors that originate in either the cytosolic mevalonate (MVA) pathway or 
the plastidic 2C-methyl erythritol 4-phosphate (MEP) pathway (Fig. 1). Labeling studies in M. truncatula ${ }^{8}$ and other species show that production of different classes of terpenes can be shared via crosstalk between the MVA and MEP pathways. Therefore, understanding the regulation of volatile terpene biosynthesis is important for the development of novel strategies of insect-pest control based on emission profiles.

Volatile profiles of $M$. truncatula plants damaged by a chewing insect (the lepidopteran Spodoptera littoralis) or a piercing-sucking insect (the aphid Tetranychus urticae) differ depending on the feeding style of the herbivore, with a greater number of compounds detected in headspace of caterpillar-damaged plants. ${ }^{9}$

At the level of gene expression in M. truncatula, both herbivory and salivary factors of the beet armyworm, Spodoptera exigua, caused a decrease in transcript accumulation of some genes encoding early steps in terpenoid biosynthesis. ${ }^{10}$ Transcripts of MtDxs 1, MtDxrl and MtHmgl were significantly reduced in leaf tissue following $S$. exigua herbivory. ${ }^{10}$ However, transcripts encoding later steps, such as those for the sesquiterpene syntase gene MtTps1, were highly induced by mechanical and insect damage. ${ }^{10,11}$ It appears that early steps in the terpene pathways leading to isopentenyl diphospate (IPP) synthesis can be regulated via several mechanisms, including both post-transcriptional and post-translational means.

Exogenous application of the phytohormone jasmonic acid (JA) on foliar tissues causes an increase in transcript levels of genes directly responsible for sesquiterpene and diterpene synthesis (MtTps1 and MtTps3) after $24 \mathrm{~h}^{8}{ }^{8}$ However, JA does not cause an increase in the transcripts for some early steps of terpene synthesis, namely MtDXS1 and MtHMGR $1 .{ }^{8}$ In addition, exogenous application on foliar tissues of 1-aminocyclopropane1-carboxylic acid (ACC), the ethylene precursor, does not increase transcript accumulation of any of the genes studied. However, combined application of JA + ACC causes an increase of MtTps5 after 24 h. $^{8}$

Terpene synthases are a class of enzymes where individual members have the rare feature of being able to take the same precursor(s) and fold it into multiple configurations. This is evident in MtTps3, where the enzyme converts the precursor farnesyl diphosphate (FPP) to the sesquiterpene nerolidol, but also uses the precursor geranylgeranyl diphosphate (GPP) to produce the diterpene geranyllinalool. ${ }^{8}$ Even more striking is the ability of MtTps 5 to utilize a single substrate, FPP, to produce a profile of at least 15 sesquiterpenes. ${ }^{8}$

We recently characterized the function of a monoterpene synthase previously annotated as $M t T p s 4,{ }^{11}$ and demonstrated that functional expression of this gene, now designated $M t E B O S$, in $E$. coli yielded an active enzyme which converts GPP into $(E)$ - $\beta$-ocimene as its major product. ${ }^{12}$ The monoterpene $(E)-\beta$ ocimene is a volatile compound released in response to herbivory by a range of plant species including cucumber, apple, lima bean, cotton and corn. ${ }^{13}$ When FPP was used as a substrate, we did not observe measureable levels of any volatile product, indicating that MtEBOS most likely does not produce a sesquiterpene product and functions specifically in plastids to produce monoterpenes.
In $M$. truncatula leaves $M t E B O S$ is inducible by lepidopteran oral secretions, lepidopteran herbivory and exogenous application of JA. Treatment of leaves with caterpillar regurgitant results in a significantly higher induction of $M t E B O S$ compared to mechanical damage, providing a strong indication that plants recognize these oral factors and respond by upregulating the latter steps in the terpene synthase pathway. ${ }^{10,11}$ We also showed that this gene is not inducible in leaf tissue by ACC which agrees with a previous report suggesting that ACC alone does not act as a signal to induce terpene synthases. ${ }^{8}$ Low-level constitutive expression of $M t E B O S$ was observed in undamaged foliar tissue, in conjunction with low levels of volatile $(E)$ - $\beta$-ocimene released from undamaged plants. In addition, two-fold higher levels of ocimene were found in volatile blends of $M$. truncatula in response to $S$. exigua feeding. In the legume Lotus japonicus, higher levels of $(E)$ - $\beta$-ocimene were detected in headspace of spider mite-infested plants, suggesting that an arthropod with a different feeding style from caterpillars can also cause release of this same monoterpene. ${ }^{14}$ It was also suggested that synthesis of $(E)$ - $\beta$-ocimene could be upregulated by pathogen infection in L. japonicus. ${ }^{14}$ Not surprisingly, amino acid residue comparison of MtEBOS with other terpene synthases demonstrates a high level of sequence conservation with $(E)$ - $\beta$-ocimene synthase of L. japonicus (67\% identity and $81 \%$ similarity). Expression analysis of $L j E B O S$ shows that this gene is upregulated by spider mite infestation and correlates with an increase of $(E)-\beta$ ocimene release. ${ }^{14}$ This is in agreement with our results, which show a clear induction of $M t E B O S$ transcript accumulation that correlates with release of the volatile end-product. Furthermore, in undamaged plants low levels of transcripts are accompanied by low levels of volatile end products. Taken together, these data all point to control of this final step of volatile terpene synthesis being regulated at the level of transcript induction.

When expressed and assayed in E. coli, MtEBOS synthesizes two minor products in addition to $(E)-\beta$-ocimene $(97.5 \%$ of total product), namely myrcene $(0.5 \%)$ and $(Z)-\beta$-ocimene $(2 \%)$. This result is reminiscent of the activity of the Arabidopsis AtTPS10-encoded enzyme, which produces both $(E)$ - $\beta$-ocimene and myrcene when expressed in E. coli. ${ }^{15}$ Thus, the newly characterized MtEBOS enzyme functions like many plant terpene synthases, in having the capacity to synthesize multiple end-products from a single substrate.

Our study provides a clear demonstration that caterpillar herbivory on $M$. truncatula induces $(E)$ - $\beta$-ocimene volatile emission, and that this process is primarily regulated by increasing expression of the gene which is directly responsible for its biosynthesis. If one considers expression of MtEBOS along with expression patterns of similar genes in related species, it is apparent that synthesis and release of volatile terpenes are regulated in very similar ways across plant families.

\section{References}

1. Kessler A, Baldwin IT. Defensive function of herbivore-induced plant volatile emissions in nature. Science 2001; 291:2141-4.

2. Baldwin IT, Preston CA. The eco-physiological complexity of plant responses to insect herbivores. Planta 1999; 208:137-45.

3. Pichersky E, Noel JP, Dudareva N. Biosynthesis of plant volatiles: Nature's diversity and ingenuity. Science 2006; 311:808-11. 
4. Dudareva N, Pichersky E, Gershenzon J. Biochemistry of plant volatiles. Plant Physiol 2004; 135:1893-902.

5. De Moraes CM, Lewis WJ, Paré PW, Alborn HT, Tumlinson JH. Herbivore-infested plants selectively attract parasitoids. Nature 1998; 393:570-3.

6. Takabayashi J, Takahashi S, Dicke M, Posthumus MA. Developmental stage of herbivore Pseudaletia separata affects production of herbivore-induced synomone by corn plants. J Chem Ecol 1995; 21:273-87.

7. Walling LL. The myriad plant responses to herbivores. J Plant Growth Regul 2000; 19:195-216

8. Arimura G, Garms S, Maffei M, Bossi S, Schulze B, Leitner M, et al. Herbivore-induced terpenoid emission in Medicago truncatula: Concerted action of jasmonate, ethylene and calcium signaling. Planta 2008; 227:453-64.

9. Leitner M, Boland W, Mithöfer A. Direct and indirect defences induced by piercingsucking and chewing herbivores in Medicago truncatula. New Phytol 2005; 167:597606.

10. Bede JC, Musser RO, Felton GW, Korth KL. Caterpillar herbivory and salivary enzymes decrease transcript levels of Medicago truncatula genes encoding early enzymes in terpenoid biosynthesis. Plant Mol Biol 2006; 60:519-31.

11. Gomez SK, Cox MM, Bede JC, Inoue KK, Alborn HT, Tumlinson JH, Korth KL. Lepidopteran herbivory and oral factors induce transcripts encoding novel terpene synthases in Medicago truncatula. Arch Insect Biochem Physiol 2005; 58:114-27.

12. Navia-Giné WG, Yuan JS, Mauromoustakos A, Murphy JB, Chen F, Korth KL. Medicago truncatula (E)- $\beta$-ocimene synthase is induced by insect herbivory with corresponding increases in emission of volatile ocimene. Plant Physiol Biochem 2009; 47:416-25.

13. Paré PW, Tumlinson JH. Plant volatiles as a defense against insect herbivores. Plant Physiol 1999; 121:325-31.

14. Arimura G, Ozawa R, Kugimiya S, Takabayashi J, Bohlmann J. Herbivore-induced defense response in a model legume. Two-spotted spider mites induce emission of $(E)-\beta$ ocimene and transcript accumulation of $(E)-\beta$-ocimene synthase in Lotus japonicus. Plant Physiol 2004; 135:1976-83.

15. Bohlmann J, Martin D, Oldham NJ, Gershenzon J. Terpenoid secondary metabolism in Arabidopsis thaliana: cDNA cloning, characterization and functional expression of a myrcene/(E)- $\beta$-ocimene synthase. Arch Biochem Biophys 2000; 375:261-9. 